\title{
Self-consistent 2D Compact Model for Nanoscale Double Gate MOSFETs
}

\author{
S. Kolberg ${ }^{1}$, T.A. Fjeldly ${ }^{2}$, and B. Iñiguez ${ }^{3}$ \\ ${ }^{1,2}$ UniK - University Graduate Center and Norwegian University of Science \\ and Technology, N-2027 Kjeller, Norway \\ \{kolberg, torfj\}@unik.no \\ ${ }^{3}$ Universitat Rovira i Virgili (URV), \\ Tarragona, E-43001, Spain \\ benjamin.iniguez@urv. net
}

\begin{abstract}
D modeling results of the electrostatics and the drain current in nanoscale DG MOSFETs are presented. The modeling of the 2D capacitive coupling within the device is based on the conformal mapping technique. In moderate above-threshold conditions, we obtain self-consistent results, which are in excellent agreement with numerical simulations.
\end{abstract}

\section{Introduction}

In nanoscale double gate (DG) MOSFETs, the electron barrier topology is a critical factor when determining conduction paths and currents in the device. A prerequisite to obtaining a precise description of such devices is to include two-dimensional (2D) effects in the models, based on a self-consistent solution of the $2 \mathrm{D}$ field pattern in the device. In such an approach, short-channel effects and scaling properties will be intrinsic to the model which, accordingly, will require only a minimal parameter set of clear physical origin.

The basic modeling problem is to obtain an analytical or semi-analytical solution of a 2D Poisson's equation where the four contacts (source, drain and the two gates) and the dielectric gaps define the boundary conditions. According to the superposition principle, Poisson's equation can be separated into a 2D Laplace equation for the capacitive coupling and the remainder involves the potential distribution established by the body charges [1 - 10]. The latter can usually be treated by simplifying considerations.

The Laplace problem for the DG MOSFET can be solved in different ways. One possibility is to perform a full Fourier expansion of the potential or by using a loworder truncation [1-4]. A corresponding procedure by means of expansion in Bessel functions has been used for cylindrical surrounding gate transistors [5]. An alternative approach is to apply the conformal mapping technique [6], which was first used for classical, long-channel MOSFETs [7]. Later, the technique was enhanced and applied to sub-100 nm devices [8] and to the subthreshold regime of undoped, nanoscale DG MOSFETs $[9,10]$. Here, we present new modeling results based on this technique applied to a wider range of operation. 
In Section 2, we discuss the conformal mapping as applied to the Laplaces problem in DG MOSFETs. In Section 3, we present a classical analysis of the self-consistent electrostatics of the device at gate voltages where the concentration of free electrons is significant.

The specific device considered is assumed to have a gate length of $L=25 \mathrm{~nm}$, a silicon thickness of $t_{S i}=12 \mathrm{~nm}$, a p-type body doping of $N_{a}=10^{15} \mathrm{~cm}-3$, an aluminum metal gate, and a high- $k$ gate insulator (nitrated Si-oxide) with a relative permittivity of 7 and a thickness of $t_{o x}=1.6 \mathrm{~nm}$ [11]. In such a small device volume, the depletion charge can be neglected. Because of the small dimensions of this device, the drain current will have the character of both drift-diffusion and ballistic transport. However, we only consider the drift-diffusion formalism since it allows us to make comparisons with numerical calculations using the Atlas simulator from Silvaco.

\section{Conformal Mapping and Capacitive Coupling}

The method of conformal mapping is applied since the solution of the Laplace equation is more easily derived in the new plane into which the device is mapped. This solution is then mapped back to the normal plane using a mapping function for the coordinates between the two planes.

\subsection{Conformal Mapping}

The device body described in the normal $(x, y)$-plane, as depicted in Fig. 1 (with 2D equipotential lines indicated), is mapped into the upper half of a complex $(u, i v)$ plane.

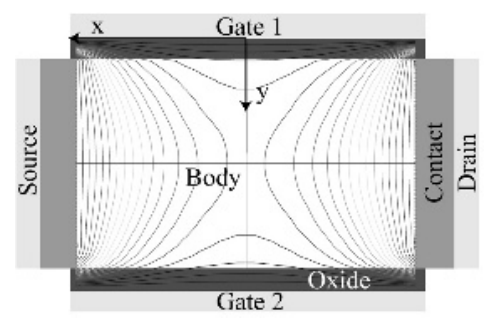

Fig. 1. Schematic view of the DG MOSFET device structure indicating the 2D equipotential lines associated with capacitive coupling between the contacts

The boundary of the body is mapped into the real $u$-axis [9], as shown in Fig. 2. The $i v$-axis represents the gate-to-gate symmetry line through the body center. To simplify the discussion, we replace the insulator of thickness $t_{o x}$ by an electrostatically equivalent silicon layer of thickness $t_{o x}^{\prime}=t_{o x} \varepsilon_{s} / \varepsilon_{o x}$, where $\varepsilon_{s}$ and $\varepsilon_{o x}$ are the permittivities of the silicon and the insulator, respectively. Laplace's equation is then considered for this extended body whose boundary is defined by the inner surfaces of the gate electrodes, the source, the drain, and the insulator gaps in the four corners between the contacts. In the strongly doped source and drain contacts, the depletion widths will be 


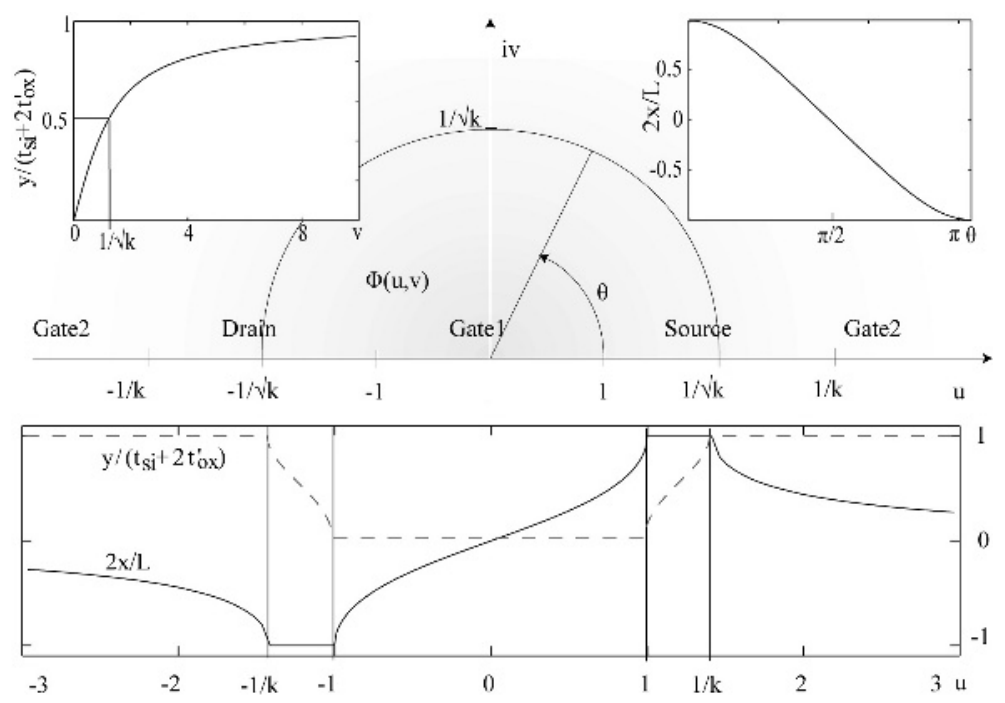

Fig. 2. The body of the DG MOSFET mapped into the upper half of the $(u, i v)$-plane. The insets show the mapping functions for the $u$-axis (lower), the $i v$-axis (upper left) and the circle with radius $1 / \sqrt{ } k$. These represent the boundary, the gate-to-gate symmetry line, and the source-todrain symmetry line, respectively.

small compared to the body dimensions and can be neglected, although the potential drops within this region should be counted.

The mapping of the boundary is defined by the following Schwartz-Christoffel transformation $[5,9,10]$ :

$$
z=x+i y=\frac{L}{2} \frac{F(k, w)}{K(k)} \quad \text { where } \quad F(k, u)=\int_{0}^{w} \frac{d w^{\prime}}{\sqrt{\left(1-w^{\prime 2}\right)\left(1-k^{2} w^{\prime 2}\right)}}
$$

Here, $F(k, u+i v)$ is the elliptic integral and $K(k) \equiv F(k, 1)$ is the complete elliptic integral, both of the first kind. The modulus $k$ is a constant between 0 and 1 determined by the geometric ratio $L /\left(t_{s i}+2 t^{\prime}{ }_{o x}\right)$. For real arguments in the standard range $0 \leq u \leq 1$, ample approximate expressions, series expansions, and iteration routines exist for $F(k, u) . F(k, u+i v)$ can also be expressed in terms of the standard elliptic integral for some other values of the argument, both real and complex. In addition, routines exist for calculating the values for general complex arguments $w$.

Note that in Fig. 2, $u=0$ corresponding to $x=0$ defines the middle point on the upper gate contact (Gate 1). The four corners of the body map to $u= \pm 1$ and $u= \pm 1 / k$. The middle point in the lower gate contact (Gate 2) is at $u= \pm \infty$ or $v=\infty$.

For the boundary, the mapping functions are given by the following expressions in terms of the standard elliptic integral (note that $F(k,-u)=-F(k, u)$ ).

Gate1:

$$
x=\frac{L}{2} \frac{F(k, u)}{K(k)}, \quad y=0
$$


S and D:

$$
x= \pm \frac{L}{2}, \quad y=\left(t_{S i}+2 t^{\prime}{ }_{o x}\right)\left[1-F\left(\sqrt{1-k^{2}}, \sqrt{\frac{1-k^{2} u^{2}}{1-k^{2}}}\right) / K\left(\sqrt{1-k^{2}}\right)\right]
$$

Gate2:

$$
x=\frac{L}{2} F\left(k, \frac{1}{k u}\right) / K(k), \quad y=t_{S i}+2 t_{o x}^{\prime}
$$

For the present device, where $k=0.4278$, the mapping function for the boundary is shown in the lower part of Fig. 2. The gate-to-gate symmetry line, which corresponds to the imaginary axis in the $w$-plane, has the following mapping function [10]:

$$
\text { G1-G2 sym. line: } \quad x=0, \quad y=\left(t_{S i}+2 t^{\prime}{ }_{o x}\right) F\left(\sqrt{1-k^{2}}, \frac{v}{\sqrt{1+v^{2}}}\right) / K\left(\sqrt{1-k^{2}}\right)
$$

Similarly, the source-to-drain symmetry line maps into a circle of radius $1 / \sqrt{ } k$ about the origin of the $w$-plane as follows,

$$
\text { S-D sym. line: } \quad x=\frac{L}{2} F\left(\frac{2 \sqrt{k}}{1+k}, \cos (\theta)\right) / K\left(\frac{2 \sqrt{k}}{1+k}\right), \quad y=\frac{t_{S i}+t^{\prime}{ }_{o x}}{2}
$$

where $\theta$ is the angle measured anticlockwise from the positive $u$-axis. The mapping functions for these two symmetry lines are shown in the upper left and right insets of Fig. 2, respectively.

Once the potential distribution has been calculated in the $(u, i v)$-plane, it can be mapped back into the $(x$, iy)-plane using the above expressions.

\subsection{Capacitive Coupling}

The potential distribution throughout the body can generally be expressed as [6]

$$
\varphi(u, v)=\frac{v}{\pi} \int_{-\infty}^{\infty} \frac{\varphi\left(u^{\prime}\right)}{\left(u-u^{\prime}\right)^{2}+v^{2}} d u^{\prime}
$$

where $\varphi\left(u^{\prime}\right)$ is the electrostatic potential along the boundary, i.e. for all values of $u^{\prime}$, and the integral runs over the entire boundary. The major contributions to this integral come from the four equipotential contacts and minor terms come from the insulator at the four corners. In the limit of zero insulator thickness, Eq. (7) results in the following analytical expression for the potential distribution in the $w$-plane [10],

$$
\varphi(u, v)=\frac{1}{\pi}\left\{\begin{array}{l}
\left(V_{G S 2}-V_{F B}\right)\left[\pi-\tan ^{-1}\left(\frac{1-k u}{k v}\right)-\tan ^{-1}\left(\frac{1+k u}{k v}\right)\right]+\left(V_{G S 1}-V_{F B}\right) \times \\
\left.v\left(\frac{1-u}{v}\right)+\tan ^{-1}\left(\frac{1+u}{v}\right)\right]+V_{b i}\left[\tan ^{-1}\left(\frac{1-k u}{k v}\right)-\tan ^{-1}\left(\frac{1-u}{v}\right)\right] \\
+\left(V_{b i}+V_{D S}\right)\left[\tan ^{-1}\left(\frac{1+k u}{k v}\right)-\tan ^{-1}\left(\frac{1+u}{v}\right)\right]
\end{array}\right\}
$$

In our calculations, the effects of a finite insulator thickness are included. $V_{G S 1}$ and $V_{G S 2}$ are the potentials of Gate 1 and Gate 2, respectively, referred to the source contact, $V_{F B}$ is the flat-band voltage for the gates and the silicon body, $V_{b i}$ is 
the built-in voltage of the source and drain, and $V_{D S}$ is the drain-source voltage. Along the two symmetry lines, Eq. (8) simplifies somewhat. Moreover, the mapping functions along these lines can be expressed in terms of the unity-range elliptic integrals.

The potential distribution according to Eq. (8) will dominate the behavior of the device in the subthreshold regime. Using the general mapping function of Eq. (1), the potential profile can now be transformed back to the $z$-plane. Fig. 3 shows $\varphi(x, y)$ for the present device in subthreshold using $V_{G S 1}=V_{G S 2}=-0.45 \mathrm{~V}$ and $V_{D S}=0.5 \mathrm{~V}$.

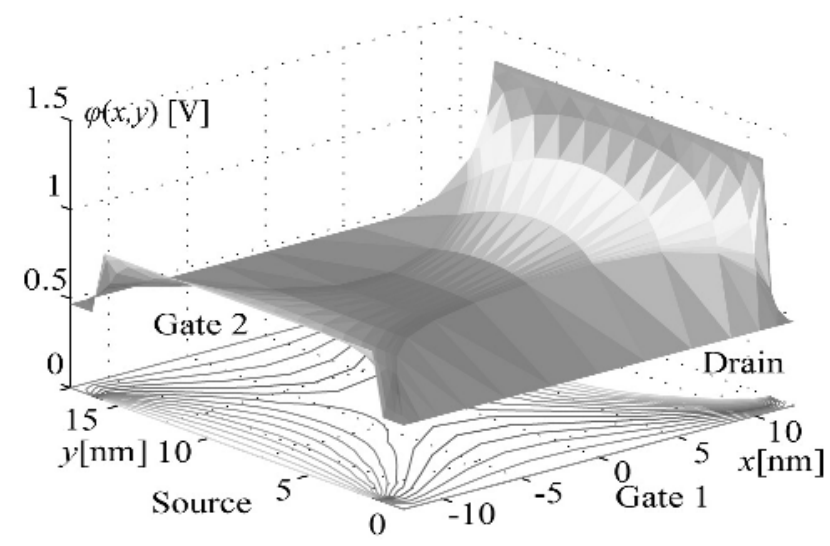

Fig. 3. Potential distribution over the extended body at subthreshold condition $\left(V_{D S}=0.5 \mathrm{~V}\right.$ and $\left.V_{G S 1}=V_{G S 2}=-0.45 \mathrm{~V}\right)$ calculated from Eq. (8) and mapped to the $(x, y)$-plane using the mapping functions discussed in Section 2.1

We note that the potential distribution has a saddle point near the device center, corresponding to the minimum barrier energy for electron conduction between source and drain. With increasing drain voltage, the barrier minimum is steadily lowered and shifted from the device center towards the source. This drain-induced barrier lowering (DIBL) is intrinsic to the present formalism as expressed in Eq. (8) [9,10]. An excellent agreement between the present model and numerical calculations using the Atlas device simulator has been demonstrated [10].

With increasing gate voltage, the barrier gate-to-gate energy profile is lowered and flattens. Eventually, the barrier minimum shifts to the silicon-insulator interfaces. When we approach this regime, the induced electron density will be sufficiently high to significantly influence the device electrostatics, requiring a self-consistent analysis (see Section 3).

The device threshold voltage $V_{T}$ can be defined in several ways, for example, in terms of a minimum current level, a minimum electron sheet density, or, as is usually done for the classical MOSFET, as the gate bias that causes a band bending by twice the silicon body Fermi potential at the barrier minimum. Using the latter definition, we find from the potential distribution of Eq. (8) that $V_{T}=-0.47 \mathrm{~V}$ for symmetric gates and zero drain bias [10]. For the other definitions, $V_{T}$ will be higher. 


\section{Self-consistent Modeling in Moderate Inversion}

In moderate inversion, the contribution of the electrons to the body potential distribution is comparable to that of the capacitive coupling. We assume a classical electron distribution and consider specifically the gate-to-gate energy barrier at the middle of the device for $V_{G S 1}=V_{G S 2}$ and $V_{D S}=0 \mathrm{~V}$. The shift in position and magnitude of the barrier at finite $V_{D S}$ (DIBL-effect) is embedded in the 2D expression of Eq. (8) and therefore carries over to the calculation of the modified, self-consistent gate-to-gate barrier profile. For the drain current modeling (see Section 4), we have adopted a simplified approach where we assume that the gate-to-gate potential distribution for finite values of $V_{D S}$ retains the same, near-parabolic form as for $V_{D S}=$ $0 \mathrm{~V}$, but scaled to reflect the correct barrier minimum as dictated by the DIBL-effect.

Along the gate-to gate symmetry line, we superimpose the $1 \mathrm{D}$ potential contribution $\varphi_{1}(y)$ from the free electrons and the 2D contribution $\varphi_{2}(y)$ from the capacitive coupling to obtain the total potential

$$
\varphi(y)=\varphi_{1}(y)+\varphi_{2}(y)
$$

Classically, $\varphi_{1}(y)$ is determined by integrating twice the 1D Poisson equation for the total potential using Boltzmann statistics for the electron density inside the silicon layer. This leads to a self-consistent expression for $\varphi_{1}(y)$ in the form of an integral equation. To solve this, we approximate $\varphi(y)$ by a symmetric parabolic form with a maximum deviation $\varphi_{m}$ from its boundary value $V_{G S}-V_{F B}$. For thin devices as here, this approximation is found to agree very well with numerical simulations within the operating range considered. By adding the resulting, explicit expression for $\varphi_{1}(y)$ to $\varphi_{2}(y)$ from Eq. (8) in Eq. (9), we obtain the following implicit, algebraic equation from which the parameter $\varphi_{m}$ can easily be extracted,

$$
\begin{aligned}
\varphi_{m}= & {\left[\frac{4}{\pi} \tan ^{-1}\left(\frac{1}{\sqrt{k}}\right)-1\right]\left(V_{b i}-V_{G S}+V_{F B}\right)-\frac{q n_{i}\left(t_{S i}+2 t^{\prime}{ }_{o x}\right)^{2}}{8 \varepsilon_{s}} \exp \left(\frac{V_{G S}-V_{F B}+\varphi_{m}-\varphi_{b}}{V_{t h}}\right) } \\
& \times\left\{\operatorname{sgn}\left(\varphi_{m}\right) \sqrt{\frac{\pi V_{t h}}{\varphi_{m}}} \operatorname{erf}\left(\sqrt{\frac{\varphi_{m}}{V_{t h}}}\left(1-\frac{2 t^{\prime}{ }_{o x}}{t s i+2 t^{\prime}{ }_{o x}}\right)\right)+\frac{V_{t h}}{\varphi_{m}}\left[\exp \left(-\frac{\varphi_{m}}{V_{t h}}\left(1-\frac{2 t^{\prime}{ }_{o x}}{t s i+2 t^{\prime}{ }_{o x}}\right)^{2}\right)-1\right]\right\}
\end{aligned}
$$

Here, erf is the error function and sgn returns the sign of its argument. Fig. 4a) shows a comparison of the potential $\varphi_{m}$ versus applied $V_{G S}$ for $V_{D S}=0 \mathrm{~V}$ as calculated from Eq. (10) and simulated classically from Atlas. Note that in the model calculations, we have adjusted $V_{b i}$ to include the effects of a finite depletion width inside the source and drain. We observe an excellent agreement between the model and the simulation within the range of $V_{G S}$ considered.

\section{Drain Current Modeling}

The small dimensions of the present device indicate that the drain current will have the character of both drift-diffusion and ballistic/quasi-ballistic transport. Here, we discuss a drain current model based the classical drift-diffusion formalism. 
For this device, the barrier topography at maximum is relatively rigid, i.e., it is little affected by the drain current for a reasonable set of bias voltages within the subthreshold and moderate inversion regimes. This allows us to use the following simple, explicit drift-diffusion model for the current that relies on the shape of the barrier near its maximum [12],

$$
I_{D D}=q W \mu_{n} n_{s}(x) \frac{d V_{F}(x)}{d x} \approx q W \mu_{n} V_{t h}\left(1-e^{-V_{D S} / V_{t h}}\right) / \int_{0}^{L} \frac{d x}{n_{s o}(x)}
$$

Here, $W$ is the device width, $V_{F}(x)$ is the quasi-Fermi potential in the channel, $n_{s}(x)$ is the sheet electron density of the channel, and $n_{s o}(x)$ is the same density in the absence of drain current (constant $V_{\mathrm{F}}$ from source through the barrier). Note that with $I_{D D}$ obtained from Eq. (11), we can go back and find an estimate of the true $V_{F}(x)$. If necessary, this, in turn, can be used to derive a self-consistent expression for $I_{D D}$, where the effect of the current on the barrier profile is included.

Fig. 4b) shows a comparison between the modeled drift-diffusion current obtained from Eq. (11) and the corresponding current using the Atlas device simulator. Note that in this model calculation, we have adjusted the effective gate length slightly to account for the true path length of the carriers through the channel. Again, we observe an excellent agreement between modeled and simulated results within the range of $V_{G S}$ considered.

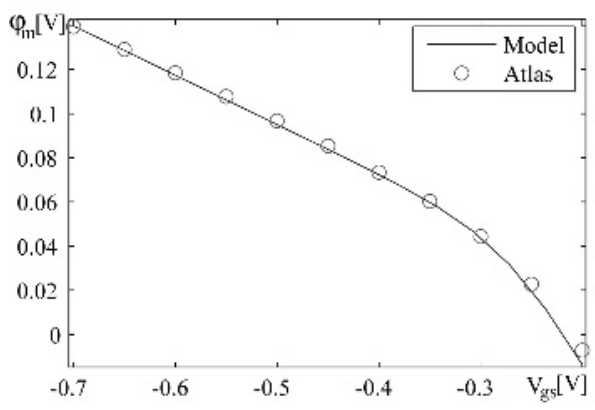

a)

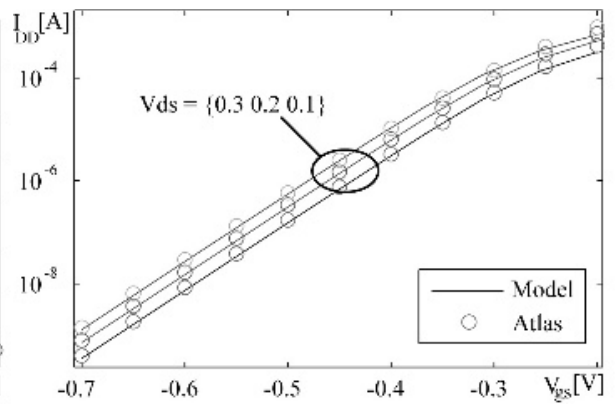

b)

Fig. 4. Comparison of $\varphi_{m}$ versus $V_{G S}$ for $V_{D S}=0 \mathrm{~V}$ (a) and drift-diffusion current versus $V_{G S}$ for $V_{D S}=0.1 \mathrm{~V}, 0.2 \mathrm{~V}$ and $0.3 \mathrm{~V}$ (b) between calculations based the present model (Eqs. (9) and (11)) (solid curves) and numerical simulations performed with Atlas (symbols)

\section{Conclusion}

We have developed a precise, compact 2D model for nanoscale DG MOSFETs for the subthreshold and the moderately strong inversion regimes of operation. The 2D modeling is based on conformal mapping techniques and a self-consistent analysis of the energy barrier topography, that include the effects of both the capacitive coupling between the contacts and the presence of electrons. Assuming a drift-diffusion transport mechanism, the drain current calculated from the present model and from 
numerical simulations (Atlas) show excellent agreement. Extensions of the model to include the strong inversion regime, quantum effects, and ballistic/quasi-ballistic transport are under way.

\section{Acknowledgement}

This work was supported by the European Commission under contract no. 506844 (SINANO) and the Norwegian Research Council under contract No. 159559/130 (SMIDA). We acknowledge the donation of TCAD tools from Silvaco.

\section{References}

1. Woo, J. S., Terrill, K.W., Vasudev, P. K.: Two-dimensional analytic modeling of very thin SOI MOSFETs. IEEE Trans. Electron Devices. vol. 37 (1990) 1999-2005

2. Frank, D. J., Taur, Y., Wong, H.-S. P.: Generalized scale length for two-dimensional effects in MOSFETs. IEEE Electron Device Letters. vol. 19 (1998) 385-387

3. Oh, S.-H., Monroe, D., Hergenrother, J. M.: Analytic Description of Short-Channel Effects in Fully-depleted Double Gate and Cylindrical, Surrounding-Gate MOSFETs. IEEE Electron Device Letters. vol. 21. no. 9 (2000) 1173-1178

4. Liang, X., Taur, Y.: A 2-D analytical solution for SCEs in the 2D MOSFET. IEEE Trans. Electron Devices. vol. 51. no. 8 (2004) 1385-1391

5. Iñíguez, B. Hamid, H. A., Jiménez, D., Roig, J.: Compact Model for Multiple Gate MOSFETs. Proc. of the Workshop on Compact Modeling, Anaheim, CA (2005) 52-57

6. Weber, E.: Electromagnetic fields, vol. 1 - Mapping of Fields. Wiley, New York (1950)

7. Klös, A., Kostka, A.: A new analytical method of solving 2D Poisson's equation in MOS devices applied to threshold voltage and subthreshold modeling. Solid-State Electronics. vol. 39 (1996) 1761-1775

8. Østhaug, J., Fjeldly, T. A., Iniguez, B.: Closed-form 2D modeling of sub-100nm MOSFETs in the subthreshold regime. J. Telecom. and Information Techn.. vol. 1/2004, (2004) 70-79

9. Kolberg, S., Fjeldly, T. A.: 2D modeling of nanoscale DG SOI MOSFETs in the subthreshold regime, accepted for publication in Journal of Computational Electronics

10. Kolberg, S., Fjeldly, T. A.: 2D Modeling of Nanoscale Double Gate SOI MOSFETs Using Conformal Mapping. Physica Scripta, accepted for publication in Physica Scripta

11. Template device for modeling and simulation defined within the European Commission Network of Excellence project SINANO (http://www.sinano.org/).

12. Fjeldly, T. A., Shur, M. S.: Threshold Voltage Modeling and the Subthreshold Regime of Operation of Short-Channel MOSFETs. IEEE Trans. Electron Devices. vol. 40, (1993) $137-145$ 